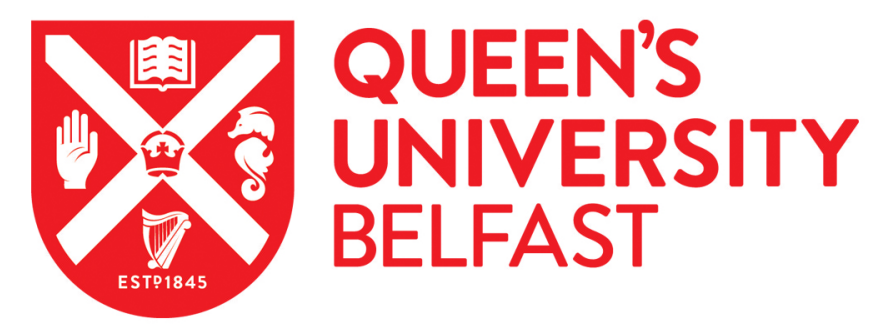

\title{
Finding the mechanism of wave energy flux damping in solar pores using numerical simulations
}

Riedl, J. M., Gilchrist-Millar, C. A., Van Doorsselaere, T., Jess, D. B., \& Grant, S. D. T. (2021). Finding the mechanism of wave energy flux damping in solar pores using numerical simulations. Astronomy \& Astrophysics, 648, [A77]. https://doi.org/10.1051/0004-6361/202040163

Published in:

Astronomy \& Astrophysics

Document Version:

Peer reviewed version

Queen's University Belfast - Research Portal:

Link to publication record in Queen's University Belfast Research Portal

Publisher rights

Copyright 2021, The European Southern Observatory.

This work is made available online in accordance with the publisher's policies. Please refer to any applicable terms of use of the publisher.

\section{General rights}

Copyright for the publications made accessible via the Queen's University Belfast Research Portal is retained by the author(s) and / or other copyright owners and it is a condition of accessing these publications that users recognise and abide by the legal requirements associated with these rights.

Take down policy

The Research Portal is Queen's institutional repository that provides access to Queen's research output. Every effort has been made to ensure that content in the Research Portal does not infringe any person's rights, or applicable UK laws. If you discover content in the Research Portal that you believe breaches copyright or violates any law, please contact openaccess@qub.ac.uk. 


\title{
Finding the mechanism of wave energy flux damping in solar pores using numerical simulations
}

\author{
J.M. Riedl ${ }^{1}$, C.A. Gilchrist-Millar ${ }^{2}$, T. Van Doorsselaere ${ }^{1}$, D.B. Jess ${ }^{2}$, and S.D.T. Grant ${ }^{2}$ \\ ${ }^{1}$ Centre for mathematical Plasma Astrophysics (CmPA), KU Leuven \\ Celestijnenlaan 200B bus 2400, 3001 Leuven, Belgium \\ e-mail: juliamaria.riedl@kuleuven. be \\ 2 Astrophysics Research Centre, School of Mathematics and Physics, Queen's University Belfast \\ Belfast, BT7 1NN, U.K.
}

Received; accepted

\begin{abstract}
Context. Solar magnetic pores are, due to their concentrated magnetic fields, suitable guides for magnetoacoustic waves. Recent observations have shown that propagating energy flux in pores is subject to strong damping with height; however, the reason is still unclear.

Aims. We investigate possible damping mechanisms numerically to explain the observations.

Methods. We performed 2D numerical magnetohydrodynamic (MHD) simulations, starting from an equilibrium model of a single pore inspired by the observed properties. Energy was inserted into the bottom of the domain via different vertical drivers with a period of 30s. Simulations were performed with both ideal MHD and non-ideal effects.

Results. While the analysis of the energy flux for ideal and non-ideal MHD simulations with a plane driver cannot reproduce the observed damping, the numerically predicted damping for a localized driver closely corresponds with the observations. The strong damping in simulations with localized driver was caused by two geometric effects, geometric spreading due to diverging field lines and lateral wave leakage.
\end{abstract}

Key words. Waves - Methods: numerical - Sun: photosphere - Sun: oscillations - sunspots - Magnetohydrodynamics (MHD)

\section{Introduction}

Solar pores are macroscopic features resembling small sunspots lacking a penumbra, but can also occur as a precursor or remnant of sunspots (Garcia de La Rosa 1987, Sobotka 2003; Thomas \& Weiss 2004). Given their nearly circular symmetry and high magnetic field concentrations, pores act as efficient wave guides + for magnetoacoustic waves, allowing wave flux to enter higher regions of the solar atmosphere (Jess et al. 2015) where the en- ergy can then be dissipated (Grant et al. 2018).

The observational evidence of waves in solar pores is vast. Photospheric sausage modes in pores were identified by, e.g. Fujimura \& Tsuneta (2009) (sausage and/or kink waves), Morton et al. (2011) (being fast waves according to Moreels et al. 2013), Dorotovič et al. (2014) (standing slow and fast waves), Grant et al. (2015) (propagating slow surface waves), Keys et al. (2018) (surface and body waves), and Gilchrist-Millar et al. (2021) (propagating slow surface and body waves, hereafter referred to as GM21). These authors all found evidence of wave periods of around 3 and/or 5 minutes, indicating the likely role of photospheric p-modes as a driver for the waves.

The propagation to the chromosphere was studied by Balthasar et al. (2000), who confirmed, by using the Vacuum Tower Telescope (VTT) on Tenerife, the presence of five-minute oscillations for the magnetic field in the deep photosphere, as seen in other observations. Using the Transition Region and Coronal Explorer (TRACE) observations, they found a peak at a period of three minutes in the chromosphere. Stangalini et al. (2011) reported longitudinal acoustic waves reaching the chro- mosphere in both three- and five-minute bands. They underline the strong connection between wave transmission and magnetic field geometry, which suggests that for pore models special attention should be paid to the definition of the magnetic field, as also suggested by Jess et al. (2013).

However, how far waves in solar pores propagate into higher layers of the solar atmosphere is still unclear. Khomenko \& Collados (2006) conducted numerical simulations of waves in a small sunspot; they used a localized wave source to study wave propagation, refraction, and mode conversion. They found that due to the vertical and horizontal stratification of the Alfvén speed, (low $\beta$ ) fast waves are refracted in the chromosphere back down to the photosphere, while slow modes continue propagating up. Recent simulations of a chromospheric resonance layer above a sunspot done by Felipe et al. (2020) show that actual wave propagation only takes place between the photosphere and chromosphere. A chromospheric resonance layer was previously also simulated by Botha et al. (2011) and observed by Jess et al. (2020). On the other hand, Riedl et al. (2019) showed in concentrated flux tubes that plane waves are converted to tube (sausage and kink) waves that are able to propagate to the corona since the tube structure greatly affects the wave propagation (Cally \& Khomenko 2019, Khomenko \& Cally 2019).

Grant et al. (2015), and more recently GM21, measured wave energy throughout the lower atmosphere of solar pores, and indeed report significant energy flux damping as a function of height. Analytic calculations of Yu et al. (2017) show that the observed damping could at least be partly explained by resonant damping of slow sausage waves. Although they find that 
this damping mechanism is stronger than previously expected, the numerical studies of Chen et al. (2018), validated by analytic calculations of Geeraerts et al. (2020), show that damping due to electrical resisitvity is much more potent than that due to resonant absorption. However, this alone is not enough to account for the damping. Flux could also be lost due to leaky tube waves (Cally 1986). Leaking waves had already been observed by Stangalini et al. (2011) and Morton et al. (2012). Grant et al. (2015) mentioned that part of the waves in their observations are reflected, which fits the simulations of Khomenko \& Collados (2006), and that mode conversion (Cally 2001; Bogdan et al. 2003) might play a role. Frequency dependent damping for slow magnetoacousic waves in sunspot umbrae is discussed by $\mathrm{Kr}-$ ishna Prasad et al. (2017), who find that higher frequencies are damped more strongly. The authors suspect this behavior occurs due to radiative and/or conductive losses.

Another important factor to consider is the cutoff frequency present in stratified media (Lamb 1909). Acoustic waves with lower frequencies than the cutoff frequency cannot propagate, but are evanescent standing waves. This effect can be used to determine the cutoff frequency of the solar atmosphere (Felipe et al. 2018), which indicates that five-minute waves like those observed by GM21 should be below the cutoff. However, the phase lag and propagation speed between different heights suggest that the observed waves in GM21 are indeed propagating as evanescent waves should not show any phase differences (Carlsson \& Stein 1997). On the other hand, the picture is not completely black and white. Centeno et al. (2006) summarized the effects of the cutoff frequency; when radiative losses are taken into account, they find that there is no clear value for the cutoff frequency, but a transition between mainly evanescent and mainly propagating waves. Therefore, it is possible that the waves in GM21 are partly subject to the cutoff, which could account for at least part of the observed damping. For the sake of this study, however, we assume the waves to be $100 \%$ propagating.

In this paper we aim to expand our understanding of the damping mechanisms in solar pores by explaining the observed damping with simple two-dimensional (2D) numerical simulations, using a model inspired by the observational parameters obtained by GM21 for their pore 3. We insert propagating waves at the bottom of the domain with a vertical velocity driver with a frequency above the cutoff frequency, and study the resulting wave energy flux with height in comparison with the data from GM21 for different setups. In Section 2 we briefly reiterate the most important points of GM21 before introducing the model, the numerical setup, and the approach for calculating the wave energy flux. The results, distinguished by driver location, are presented in Section 3 and thoroughly discussed in Section 4. A short discussion about the case of a driver with frequency lower than the cutoff frequency is presented in Appendix A

\section{Methods}

\subsection{Observations}

The model developed in this work is inspired by the observations detailed in GM21, who utilized data obtained by the Facility Infrared Spectropolarimeter (FIRS; Jaeggli et al. 2010) based at the National Science Foundation's Dunn Solar Telescope (DST), Sacramento Peak, New Mexico. The FIRS data consist of sitand-stare slit-based spectropolarimetric observations of the decaying active region NOAA 12564, which was captured between 14:09 - 15:59 UT on 2016 July 12 in the Si I $10827 \AA$ spectral

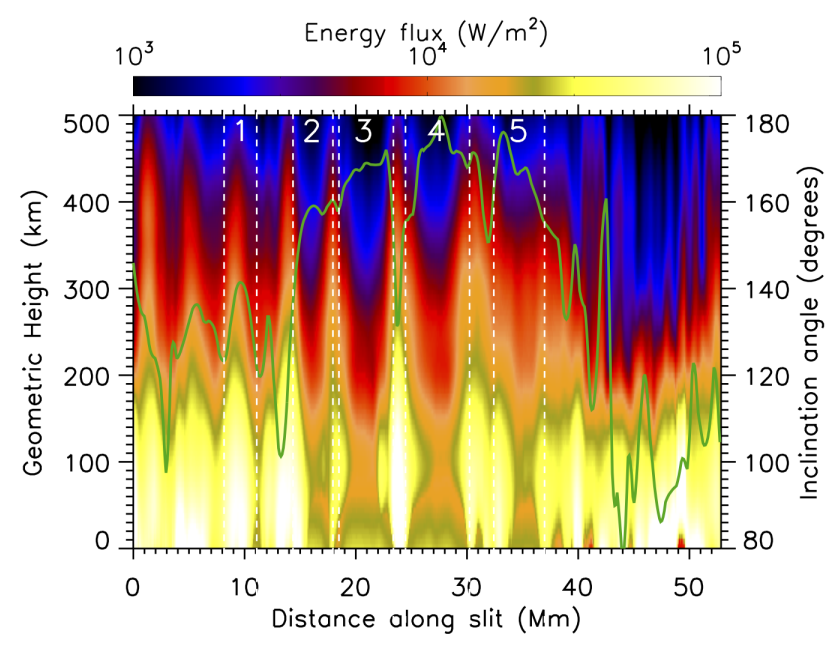

Fig. 1. Energy flux across all five observed pores as a function of height. The color scale is logarithmic. Pore boundaries are shown as white dashed lines. The green solid line shows the inclination angle of the magnetic field. From GM21

line. The observations acquired contain a set of five solar pores that were positioned along a unique straight-line configuration. To cover all pores in a single FIRS exposure, the DST coude table was rotated so the spectrograph slit passed through the center of each photospheric pore boundary.

An examination of the photospheric Si I $10827 \AA$ line bisector velocities showed periods on the order of five minutes across all pore structures. Through spectropolarimetric inversions using the Stokes Inversion based on Response functions (SIR; Ruiz Cobo \& del Toro Iniesta 1992) code, the local plasma densities, magnetic field strengths, and temperatures were deduced as a function of atmospheric height spanning the range $0-500 \mathrm{~km}$. The central pore (pore 3 in GM21) exhibited the best signal-tonoise ratio, and so was selected for comparison with the present theoretical work.

For pore 3 documented by GM21, the magnetic fields were found to be close to vertical toward the pore center, with field strengths of $2400 \mathrm{G}$ and $1000 \mathrm{G}$ at atmospheric heights of 0 $\mathrm{km}$ and $500 \mathrm{~km}$, respectively. Temperatures ranged from 5000 $\mathrm{K}$ to $3500 \mathrm{~K}$ and densities spanned from $8.5 \times 10^{-4} \mathrm{~kg} \mathrm{~m}^{-3}$ to $9.8 \times 10^{-6} \mathrm{~kg} \mathrm{~m}^{-3}$ across the same height range. Parameters derived from the inversions were combined with mean square velocities to calculate energy flux estimates as a function of atmospheric height (Equation 12). The energy flux across all five pores as a function of height is displayed in Figure 1 Pore 3 was found to exhibit considerable energy damping with an average energy flux on the order of $25 \mathrm{~kW} \mathrm{~m}^{-2}$ at an atmospheric height of $100 \mathrm{~km}$, dropping to $1.5 \mathrm{~kW} \mathrm{~m}^{-2}$ at $500 \mathrm{~km}$. The damping mechanisms producing this drop in energy flux remain elusive. In addition, an increase in energy flux toward the boundaries of pore 3 indicated the presence of surface mode waves.

\subsection{Model}

In order to investigate the wave damping in solar pores with numerical simulations, we first need to create a 2D gravitationally stratified magnetohydrostatic (MHS) equilibrium atmosphere that resembles the observational data. For a MHS equlib- 
rium the following condition must be fulfilled

$\boldsymbol{\nabla} p-\frac{1}{\mu_{0}}(\boldsymbol{\nabla} \times \boldsymbol{B}) \times \boldsymbol{B}-\rho \boldsymbol{g}=0$,

where $p$ is the gas pressure, $\rho$ is the density, $\boldsymbol{B}$ is the magnetic field, $\mu_{0}$ is the magnetic permeability, and $\boldsymbol{g}$ is the gravitational acceleration.

We start by choosing the magnetic field in the $z$-direction

$B_{z}(x, z)=a(z)\left[\arctan \left(\frac{x+r(z)}{s(z)}\right)-\arctan \left(\frac{x-r(z)}{s(z)}\right)\right]+b(z)$,

which results in a bundle of strong vertical magnetic field of $a(z)$ inside the pore above the background field $b(z)$ outside the pore, resembling the observations. The parameter $r(z)$ describes the radius of the pore, while $s(z)$ is the smoothness parameter, which defines the thickness of the transition between pore and background. For the sake of simplicity, the written dependence on the vertical coordinate $(z)$ is omitted from now on for these four parameters.

The parameters defining Equation 2 are

$\begin{array}{llll}a & =0.33 G_{\text {axis }}[\mathrm{T}], & r & =\frac{2 \times 10^{5}}{\sqrt{G_{\text {axis }}}}[\mathrm{m}], \\ b & =0.05 G_{\text {side }}[\mathrm{T}], \quad s= & 0.1 r & {[\mathrm{~m}],}\end{array}$

with exponential functions approximating the observed magnetic field strength of pore 3 from GM21 at the axis of the pore $G_{\text {axis }}=$ $0.1 \exp (-z / 300000)+0.07[\mathrm{~T}]$ and the side of the pore $G_{\text {side }}=$ $0.1 \exp (-z / 300000)+0.02[\mathrm{~T}]$.

Because $\operatorname{div} \boldsymbol{B}=0$, we know that in $2 \mathrm{D}$

$\frac{\partial B_{x}}{\partial x}=-\frac{\partial B_{z}}{\partial z}$.

Therefore,

$$
\begin{aligned}
B_{x}(x, z)= & -\int \frac{\partial B_{z}}{\partial z} d x+h(z) \\
= & -\frac{d a}{d z}\left[\frac{s}{2} \ln \left(\frac{(x-r)^{2}+s^{2}}{(x+r)^{2}+s^{2}}\right)\right. \\
& \left.+(x+r) \arctan \left(\frac{x+r}{s}\right)-(x-r) \arctan \left(\frac{x-r}{s}\right)\right] \\
& -a\left[\frac{1}{2} \frac{d s}{d z} \ln \left(\frac{(x-r)^{2}+s^{2}}{(x+r)^{2}+s^{2}}\right)\right. \\
& \left.+\frac{d r}{d z} \arctan \left(\frac{x+r}{s}\right)+\frac{d r}{d z} \arctan \left(\frac{x-r}{s}\right)\right]-\frac{d b}{d z} x,
\end{aligned}
$$

where we assume that $h(z)=0$ because then the solution is antisymmetric around $x=0$.

In order to get a solution that fulfills both components of Equation 11.

$\frac{\partial^{2} p}{\partial x \partial z}=\frac{\partial^{2} p}{\partial z \partial x}$

must be true. By differentiating the $x$-component of Equation 1 with respect to $z$ and the $z$-component with respect to $x$, and combining the resulting derivatives with Equation 6, we find a constraint for the density,

$$
\begin{aligned}
\frac{\partial \rho}{\partial x}=\frac{1}{\mu_{0} g}[ & \frac{\partial B_{x}}{\partial x}\left(\frac{\partial B_{z}}{\partial x}-\frac{\partial B_{x}}{\partial z}\right)+B_{x}\left(\frac{\partial^{2} B_{z}}{\partial x^{2}}-\frac{\partial^{2} B_{x}}{\partial z \partial x}\right) \\
& \left.+\frac{\partial B_{z}}{\partial z}\left(\frac{\partial B_{z}}{\partial x}-\frac{\partial B_{x}}{\partial z}\right)+B_{z}\left(\frac{\partial^{2} B_{z}}{\partial x \partial z}-\frac{\partial^{2} B_{x}}{\partial z^{2}}\right)\right],
\end{aligned}
$$

assuming $g=[0,-g]$ with $g=274 \mathrm{~m} / \mathrm{s}$. The density can then be obtained with

$\rho(x, z)=\int \frac{\partial \rho}{\partial x} d x+f(z)$.

The function $f(z)$ is of great importance here as it defines the gravitational stratification of the density. We therefore set $f(z)$ to be equal to the average density obtained from the observations of GM21 for their pore 3. Since $\partial \rho / \partial x$ also has a dependence on $z$, we add a small constant to $\rho$ to ensure its non-negativity. Due to the complexity of Equation 8 it is solved numerically.

From the second component of Equation 1, the pressure can be calculated with

$p(x, z)=\int \frac{\partial p}{\partial z} d z+j(x)$.

As long as the pressure is symmetric around the pore axis at $x=0$, there is no need to add a function $j(x)$. However, we add a constant to ensure a positive pressure. This equation is also solved numerically.

Theoretically, the described model is in MHS equilibrium. However, numerical calculations as used in the solution of the model and in the simulation code itself are imperfect, often resulting in somewhat unstable behavior, especially when gravity is involved. Therefore, using the boundary conditions described in Section 2.3, we simulate the model without driver for a physical time of 1300 seconds to let it settle down. After this time, there are no significant changes to density, magnetic field, or pressure on a timescale compared to a few driver periods. This slightly relaxed atmosphere is then used as the initial condition for our simulations. We note, however, that even after the slight relaxation there are still significant velocities within the domain, meaning that the resulting model atmosphere has not completely settled to a MHS equilibrium.

The top panel of Figure 2 shows the vertical magnetic field component of the initial atmosphere, with field lines shown in orange. Due to the symmetry of the problem, only half of the pore is included in our model, with the pore axis being located at $x=0$. The pore itself is located on the left side of the plot, where the magnetic field is strong and mainly vertical. The deviation of the horizontal profile from the arctan-shape of Equation 2 occurs because of the equilibration process. The comparison of the model with the observations of GM21 (Figure 2 bottom) shows great similarity. It should be noted, however, that the horizontal extent of our model pore (FWHM radius $\approx 0.44 \mathrm{Mm}$ ) is smaller than the pores in the observations (radius $\approx 2.5 \mathrm{Mm}$ ). Even so, when comparing the magnetic field inclination of the model atmosphere with the field inclination of pore 3 from GM21 in the direction perpendicular to the slit (thus perpendicular to the line of five pores), while taking the different radii into account, the field inclinations also coincide quite well (see Figure 3). The plasma- $\beta$ in our model is higher than unity everywhere, with values ranging from 2 to 6.5 inside the pore and higher values up to 40 and higher outside.

Similarly, Figure 4 shows the density of the settled model and the comparison to observations, where the density structures seen appear during the equilibration process. It is immediately apparent, that the model density is much less stratified with height than the observations, even though we added the observational density as stratification in Equation 8. This is caused by the effect of $\partial \rho / \partial x$ calculated by Equation 7 already having a dependence on $z$, which in total decreases the stratification. In addition, it is also slightly decreased when the atmosphere is allowed to settle down. However, it should be noted that for the 

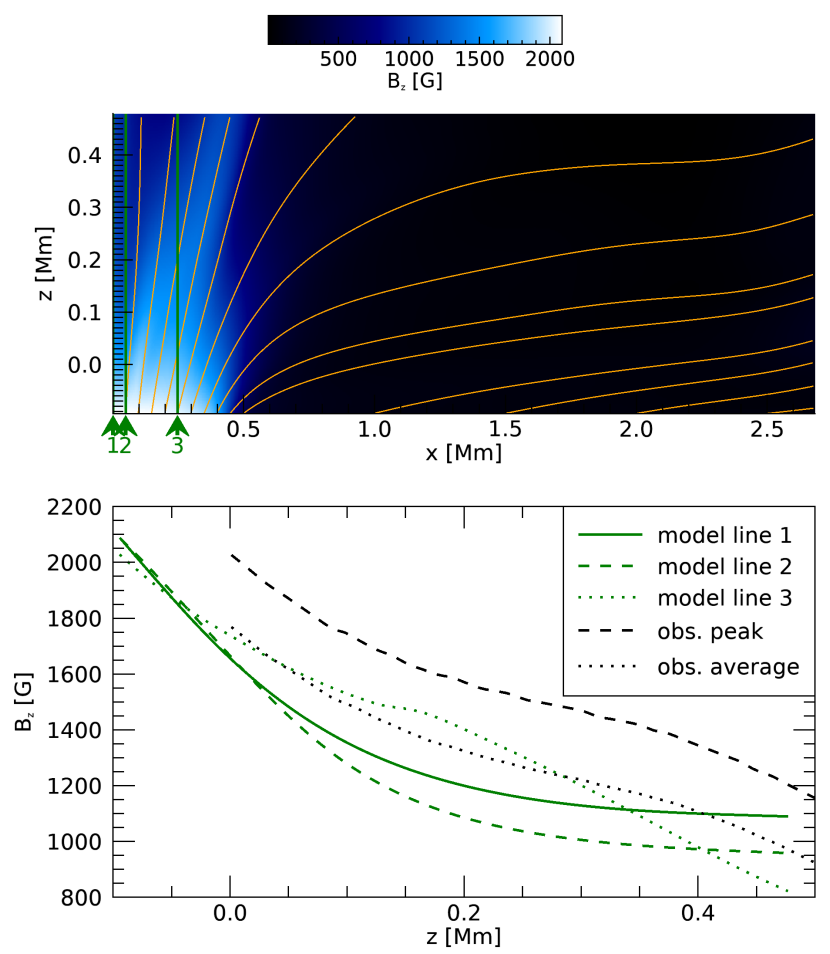

Fig. 2. Top: Vertical component of the magnetic field of the settled model atmosphere. Orange lines depict the magnetic magnetic field lines. Bottom: Comparison of the model atmosphere with the observations from GM21 of pore 3. The maximum observational value within the pore is shown by obs. peak, while the horizontal average across the whole pore is shown by obs. average. The green lines show the model values for the indicated lines in the top figure (line 1 at pore axis).

observations in GM21, the density is not a direct output of the inversions, but is instead determined through solving equations of state using inferred inversion outputs, under the assumption of hydrostatic (HS) equilibrium. This simplifying assumption is problematic in strong magnetic fields as it ignores the Lorentz force, thus providing notable uncertainties on the densities input into the model, of up to an order of magnitude (Borrero et al. 2019).

Nonetheless, the density values from GM21 are still consistent with those from semi-empirical models like that of Maltby et al. (1986), who considered a magnetized atmosphere at the center of a sunspot umbra. They also assumed a HS equilibrium; however, this assumption is valid for the center of an axially symmetric sunspot as the magnetic terms in Equation 1 vanish. Therefore, we have to assume that the observational values of the density are more reliable than the model values.

The smaller pore radius and less stratified density in our model compared to the observations are due to compromises being made when solving Equation 1 Once a non-force-free magnetic field is chosen, the density or pressure cannot be freely chosen, but only manipulated through the addition of integration constants, as can be seen in Equations 8 and 9 Therefore, in order to obtain a stable model for our simulations, certain concessions have to be made. In addition, due to the same reasons, our model also results in a plasma- $\beta>1$ inside the pore, as opposed to a low $\beta$ found by GM21 within the pores. The impact of the differences between observations and theoretical model on our results is discussed in Section 4.1 .

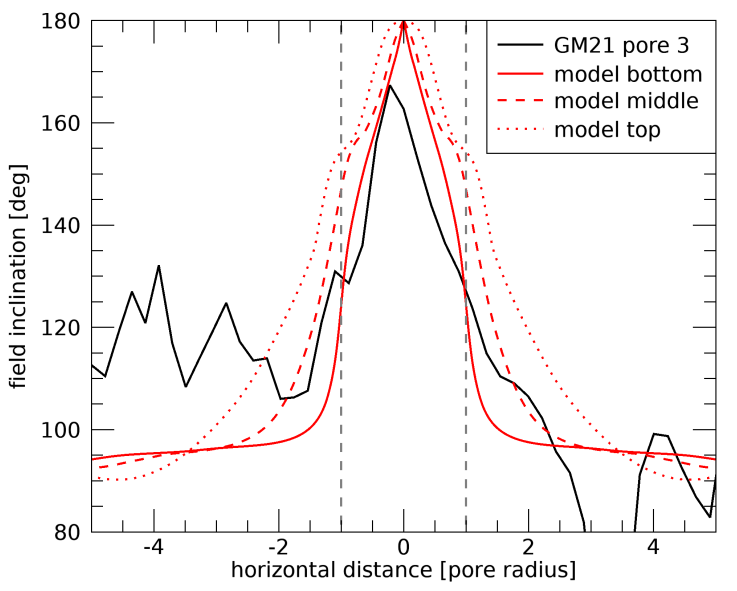

Fig. 3. Comparison of the magnetic field inclination between model (red) and observations for pore 3 of GM21 (black) as a function of horizontal distance normalized to the pore radius. The pore radius for the model was assumed to be $0.44 \mathrm{Mm}$, while the radius of the observed pore is $2.5 \mathrm{Mm}$. The observational values are taken along the line perpendicular to the slit. Model values are mirrored around $x=0$ and are shown for the bottom (solid line), middle (dashed line), and top part (dotted line) of the model. The vertical dashed gray lines show the border of the pores at $x=1$.
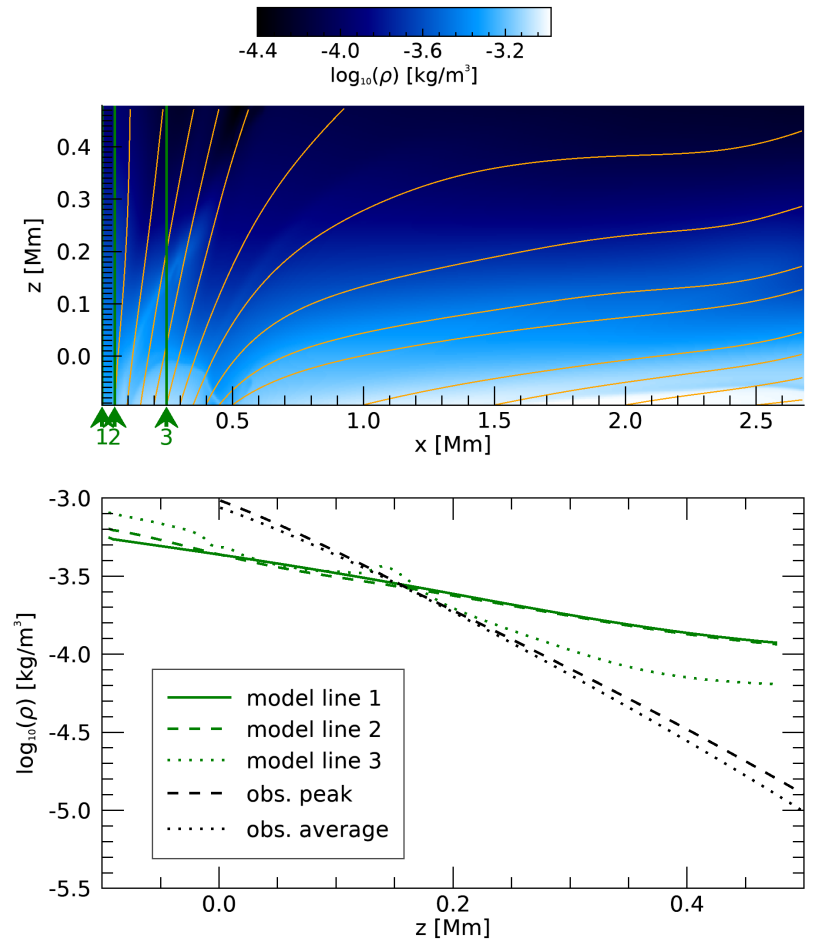

Fig. 4. Top: Logarithm of the density of the settled model atmosphere. Orange lines depict the magnetic magnetic field lines. Bottom: Comparison of the model atmosphere with the observations from GM21 of pore 3 . The maximum observational value within the pore is shown by obs. peak, while the horizontal average across the whole pore is shown by obs. average. The green lines show the model values for the indicated lines in the top figure (line 1 at pore axis).

\subsection{Numerical setup}

All our simulations are conducted using the PLUTO code (Mignone et al. 2007), which solves the magnetohydrodynamic (MHD) equations when using the respective module. Fluxes are 
computed using a linearized Roe Riemann solver, while the time step is advanced using an unsplit second-order accurate characteristic tracing method, which is less dissipative than multi-step algorithms. To deal with the inevitable occurrence of $\operatorname{div} \boldsymbol{B}$ we use the mixed hyperbolic/parabolic divergence cleaning technique of Dedner et al. (2002), which is further discussed in Mignone et al.(2010). Gravity is added using a body force with constant acceleration toward the negative $z$-direction.

Keeping a model atmosphere stable when gravity is included can often prove difficult and is highly contingent on the boundary conditions at boundaries perpendicular to the gravitational acceleration. In our case it was not possible to set fully open boundary conditions at the top boundary. We therefore expand the model atmosphere at the top to add a thick high-viscosity layer to absorb all outgoing waves, effectively having an open boundary. We use the same boundary condition for the right boundary. The viscosity is treated with an explicit time integration. Including the viscous layers the domain ranges from 0 to $3 \mathrm{Mm}$ in the $x$-direction and from -0.095 to $0.795 \mathrm{Mm}$ in the $z$ direction with $1000 \times 297$ cells, leading to a spatial resolution of $3 \mathrm{~km}$ in both directions. Excluding the viscous layers, a physical domain remains ranging from 0 to $2.68 \mathrm{Mm}$ with 894 cells in the $x$-direction and from -0.095 to $0.475 \mathrm{Mm}$ with 191 cells in the $z$ direction. Only this physical domain is used for the analysis and figures. The height of $0 \mathrm{Mm}$ is defined as the bottom of the photosphere. After settling the calculated model from Section 2.2 for $1300 \mathrm{~s}$ (defined as $t=0 \mathrm{~s}$ in the plots), the simulations are run for an additional $200 \mathrm{~s}$.

Due to the symmetry of the system our model only includes half of a solar pore, with the pore axis being located at the left boundary. Thus, the boundary conditions there are set to be reflective. At the bottom boundary we set pressure, density, and magnetic field to fixed values that fulfill the equations presented in Section 2.2 for the initial model before the equilibration. The horizontal velocity is set to 0 . For simulations without driver, the vertical velocity is set to 0 as well. When a driver is included the vertical velocity is set according to

$v_{z, \text { driver }}=A \sin \left(\frac{2 \pi}{T} t\right)$,

with the amplitude $A=160 \mathrm{~m} / \mathrm{s}$ and the period $T=30 \mathrm{~s}$. Since the driver purely perturbs the velocity, some of the driver energy immediately flows into pressure and density perturbations. Between the ghost cells (additional cells outside the computation domain to enable numerical integration) including the driver and the first cell of the domain, the root mean square of the velocity perturbation is therefore reduced to levels observed by GM21 at the bottom of the pores of about $50 \mathrm{~m} / \mathrm{s}$. This short period for the driver was chosen because a typical p-mode period of $300 \mathrm{~s}$ is close to the cutoff period in our model, leading to the formation of standing waves due to reflections. However, we want to investigate propagating waves and their damping. In addition, for longer periods the wavelength would increase accordingly, causing the resulting waves to not fit into the domain. For the sake of completeness, we also did simulations with a low-frequency driver below the cutoff frequency, and we show a crude analysis in Appendix A

For some of our simulations we include non-ideal effects like viscosity, resistivity, and thermal conduction. Those effects were added using explicit time integration, and for expected values in the photosphere $\left(R_{e}\right.$ and $R_{m}$ taken from Ossendrijver 2003). In the case of the simulations with viscosity, where viscosity was also present in the physical domain, simulations were done with exaggerated values for the viscous shear coefficient.

\subsection{Wave energy flux}

The energy flux can be calculated as (e.g., Goedbloed \& Poedts 2004)

$$
\boldsymbol{E}=-\frac{1}{\mu_{0}}(\boldsymbol{v} \times \boldsymbol{B}) \times \boldsymbol{B}+\left(\frac{\rho v^{2}}{2}+\rho \Phi+\frac{\gamma}{\gamma-1} p\right) \boldsymbol{v},
$$

where $\Phi=-g z+$ const. is the gravitational potential. The left term of Equation 11 is the Poynting flux, which is the magnetic component of the energy flux, whereas the other terms describe the hydrodynamic component. Since in our model $\beta>1$ everywhere and the driver mainly excites acoustic waves, the hydrodynamic component is dominant in our simulations.

There are still velocities up to nearly $2 \mathrm{~km} / \mathrm{s}$ within the whole physical domain or up to $\sim 350 \mathrm{~m} / \mathrm{s}$ within the pore after settling the atmosphere for $1300 \mathrm{~s}$. These velocities are higher than the driver amplitude. Thus, in addition to simulations with a driver, we also conduct simulations without a driver, allowing us to extract the effects caused by the input waves alone. This is done by subtracting all the variables of the simulations without a driver from the variables of the simulations with a driver, effectively giving us the perturbed variables

$$
\begin{array}{ll}
\rho^{\prime}=\rho_{\text {driver }}-\rho_{\text {nodriver }}, & p^{\prime}=p_{\text {driver }}-p_{\text {nodriver }}, \\
\boldsymbol{v}^{\prime}=\boldsymbol{v}_{\text {driver }}-\boldsymbol{v}_{\text {nodriver }}, & \boldsymbol{B}^{\prime}=\boldsymbol{B}_{\text {driver }}-\boldsymbol{B}_{\text {nodriver }} .
\end{array}
$$

To obtain the wave energy flux, these perturbed variables are put into Equation 11, in a process that is similar to linearization.

In GM21, on the other hand, the wave energy flux was calculated as

$$
E=\rho v_{g}\left\langle v^{2}\right\rangle
$$

with $v_{g}$ being the group speed and $\left\langle v^{2}\right\rangle$ being the mean square velocity. For our simulations, Equations 11 and 12 yield similar trends with absolute values in the same order of magnitude. Using Equation 11 facilitates a more detailed analysis, which is possible due to the much more detailed knowledge of the data in simulations compared to observations.

\section{Results}

We conducted a range of simulations, including and removing non-ideal effects, and applying differing drivers. Depending on the driver location, the results can be divided into two distinct groups, which are discussed in the following.

\subsection{Driver located at whole bottom boundary}

We applied the velocity driver described in Equation 10 on the whole bottom boundary, resulting in plane fast waves propagating upward at approximately the sound speed. A single snapshot of the vertical velocity perturbation after two driver periods is shown in Figure 5. The amplitude of the waves increases with height, as is expected due to the conservation of energy in a stratified plasma. The wave fronts are not completely horizontal, but have a jagged form at the pore location. This happens due to differing wave speeds at different locations. The vertical wave ridges visible at $x \approx 0.6 \mathrm{Mm}$ and the right boundary, and more pronounced at later times, as seen in the movie of the time sequence, are wave fronts of slow waves.

Figure 6 shows the time-averaged wave energy flux as a function of height relative to the first measure point obtained from the observations of GM21 for their pore 3. Both simulation and 


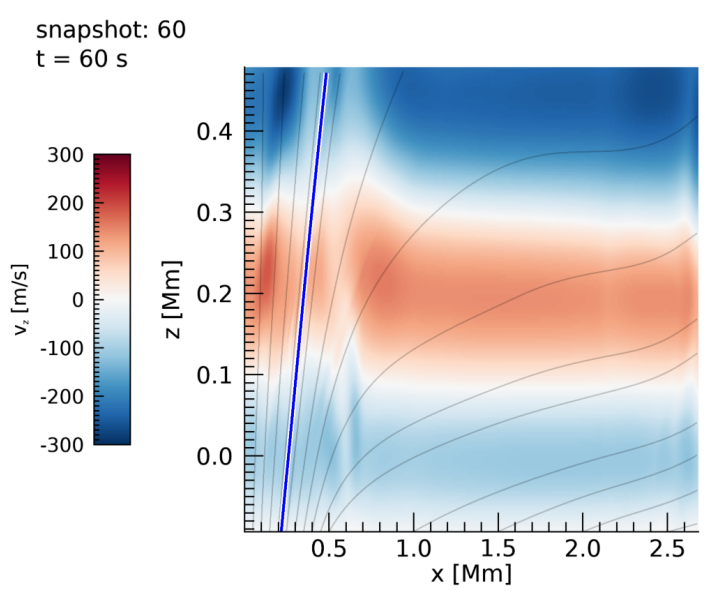

Fig. 5. Snapshot of the vertical velocity perturbation after two periods for the full driver and ideal MHD. The gray lines show magnetic field lines. The blue line highlights the field line considered for the analysis in Figure 6 The full time sequence is available as a movie online.

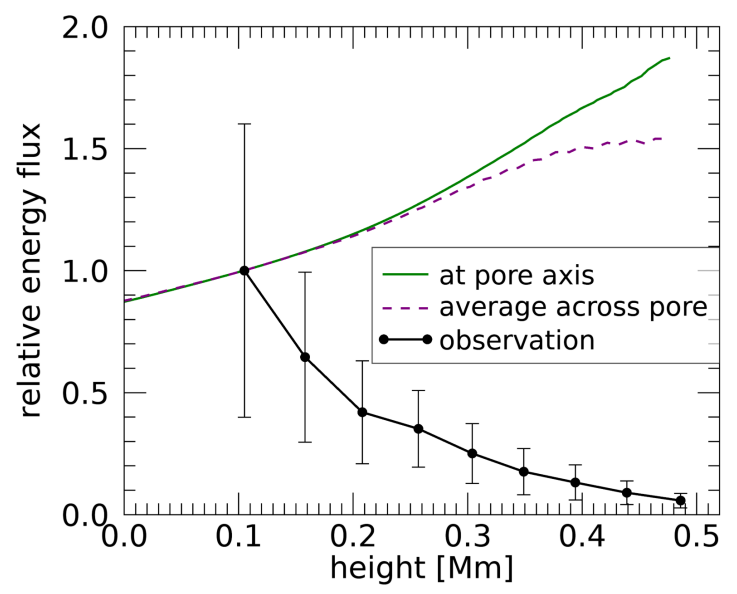

Fig. 6. Relative wave energy flux parallel to the magnetic field averaged over time as a function of height for the full driver. The solid green line shows the energy flux along the pore axis, whereas the dashed purple line shows the average flux across the pore up to the field line highlighted in Figure 5. The observational data (black line with symbols) are from pore 3 of GM21 All fluxes are normalized to the first observational data point.

observational data were normalized to the data point at $z=0.1$ $\mathrm{Mm}$. The time average of the simulation data was taken over the first period of the propagating wave. The figure shows the energy flux at the pore axis, where the magnetic field line is vertical (green line), and the flux averaged from the pore axis to the location of the field line highlighted in Figure 5 (purple dashed line). The observational data were also averaged in time and across the pore.

It is evident from Figure 6 that there is no indication of wave damping with height in our simulations; instead, the energy flux even increases with height, which could be explained by waves being refracted into the pore, as discussed in Section 4.1. The lack of damping is not only the case for ideal MHD, but also when resistivity, viscosity, or thermal conduction is included. Even for exaggerated viscosity no damping is achieved. We assume this is the case because we are studying a very narrow slab of atmosphere of a few hundred kilometers, leaving little time for non-ideal effects to affect the waves. Therefore, we fail to re-

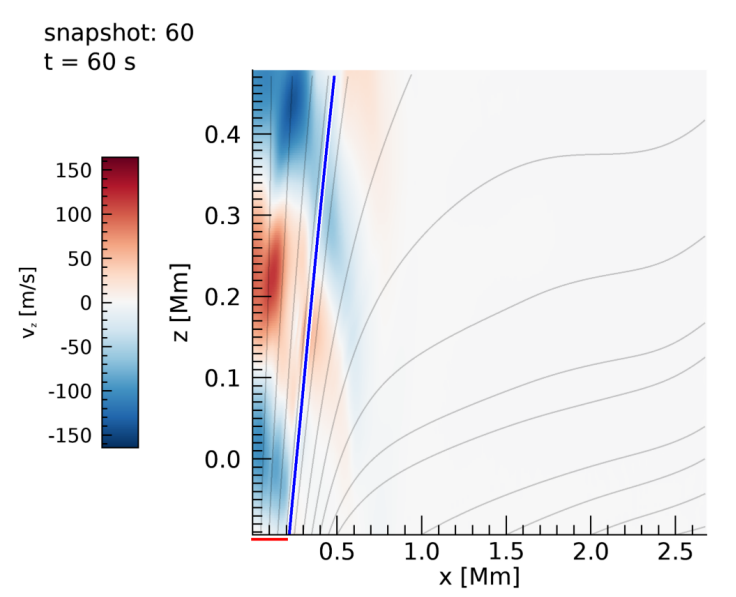

Fig. 7. Snapshot of the vertical velocity perturbation after two periods for the localized driver for ideal MHD. The gray lines show magnetic field lines. The red bar below the $x$-axis indicates the driver location. The blue line highlights the field line considered for the analysis in Figure 8 . The full time sequence is available as a movie online.

produce the observed damping with a plain driver located at the whole bottom boundary.

\subsection{Localized driver}

Solar pores are magnetic structures that do not form in the photosphere but are already present below the solar surface. As solar pores are good wave guides, it is valid to assume that only the pore itself may be driven. Numerical simulations (Cameron et al. 2007) supported by observations (Cho et al. 2013) suggest that rapid cooling within pores could lead to downflows that collide with the plasma of lower layers to produce rebounding upflows, which further motivates the assumption of a localized driver. Moreover, previous simulations (Kato et al. 2016) show that photospheric buffeting by turbulent motions lead to the efficient excitation of waves. We therefore alter our driver to a stepfunction driver that is only present in the inner part of the pore

$v_{z, \text { driver }}= \begin{cases}A \sin \left(\frac{2 \pi}{T} t\right) & x \leq 0.2 \mathrm{Mm} \\ 0 & x>0.2 \mathrm{Mm}\end{cases}$

Figure 7 shows a snapshot of the vertical velocity perturbation after two periods for the step-function driver. In contrast to the respective figure for the full driver, the wave fronts are not horizontal and the maximum amplitude is lower because the atmosphere is only driven at one location (indicated by the red bar below the $x$-axis). The blue line highlights a field line rooted slightly outside the driver location at $x=0.22 \mathrm{Mm}$. There are clearly waves present beyond this field line, suggesting that the waves do not purely propagate along the magnetic field.

If we now study the wave energy flux as a function of height for the simulation with localized driver, as shown in Figure 8 (left), it is immediately apparent that the energy flux is now strongly damped, in stark contrast to the simulations with the full driver. This sudden drop in wave energy flux with height by just changing the driver location can be explained by two geometric mechanisms: geometric spreading and lateral wave leakage.

\subsubsection{Geometric spreading}

The magnetic field lines in our model diverge with height. Therefore, if the waves were perfectly propagating along the field 


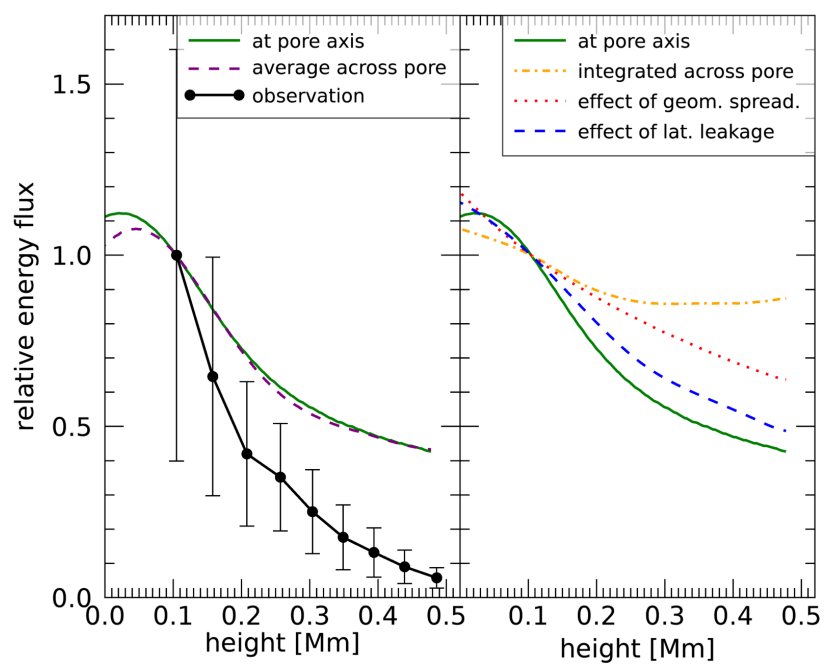

Fig. 8. Left: Relative wave energy flux parallel to the magnetic field averaged over time as a function of height for the localized driver. The solid green line shows the energy flux along the pore axis, whereas the dashed purple line shows the average flux across the pore up to the field line highlighted in Figure 7 The observational data (black line with symbols) are from pore 3 of GM21 All fluxes are normalized to the first observational data point. Right: Comparison of flux damping in the simulation with localized driver with the effects of geometric damping. The solid green line shows the same data as in the left plot for comparison. The other lines are explained in the text.

lines, the flux along a single field line, as well as the average flux at each height within the pore, would be expected to drop due to the flux being distributed across a wider area with increasing heights. The decrease in flux with height due to this mechanism is proportional to $1 / R$ in 2D geometry, where $R$ is the distance between the pore axis and a specific field line. Such a curve is shown in Figure 8 (right, dotted red line) for the field line highlighted in Figure 7. Since this curve drops substantially less with height than the wave flux, there must be another mechanism with approximately equal significance.

In addition, if only geometric spreading caused the damping, the wave flux parallel to the magnetic field integrated across the pore should be constant with height because the same total amount of flux would be contained inside the pore at all heights. This is not the case, which can be seen with the dash-dotted orange line in Figure 8, Therefore, flux must escape from the pore through its edges.

\subsubsection{Lateral leakage}

In our simulations with a localized driver, we observe waves propagating out of the solar pore, which decreases the flux inside the pore. This is the case because magnetoacoustic waves can propagate at an inclined angle with respect to the magnetic field. In a homogeneous plasma, the phase speed of fast and slow magnetoacoustic waves is (e.g., Goedbloed et al. 2019)

$v_{\mathrm{fa} / \mathrm{sl}}(\theta)=\frac{\sqrt{v_{s}^{2}+v_{A}^{2}}}{\sqrt{2}}\left[1 \pm\left(1-\frac{4 v_{c}^{2} \cos ^{2} \theta}{v_{s}^{2}+v_{A}^{2}}\right)^{1 / 2}\right]^{1 / 2}$,

where $v_{s}$ is the sound speed, $v_{A}$ the Alfvén speed, $v_{c}=v_{A} v_{S} /\left(v_{A}^{2}+\right.$ $\left.v_{s}^{2}\right)^{1 / 2}$ the cusp speed, and $\theta$ the angle between the propagation direction and the magnetic field. The positive (negative) sign is for the calculation of the phase speed of the fast (slow) wave. In a plasma where $v_{s}>v_{A}$ (approximately $\beta>1$ ), the phase speed of fast waves takes the shape of a flattened quasicircle with $v_{\mathrm{fa}}(\theta=0, \pi)=v_{s}$ in the magnetic field direction and $v_{\mathrm{fa}}(\theta=\pi / 2,3 \pi / 2)=\left(v_{A}^{2}+v_{s}^{2}\right)^{1 / 2}$ perpendicular to it. On the other hand, slow waves take the shape of double quasicircles with $v_{\mathrm{sl}}(\theta=0, \pi)=v_{A}$ in the magnetic field direction and $v_{\mathrm{sl}}(\theta=\pi / 2,3 \pi / 2)=0$ perpendicular to the magnetic field. Therefore, also for slow waves there is still a non-zero phase speed for all directions except exactly perpendicular to the magnetic field. This effect was previously used by Nakariakov \& Zimovets (2011) to explain flare ribbon propagation.

Assuming local homogeneity and utilizing Equation 13 , we can apply the Huygens-Fresnel principle to theoretically predict the locations of fast and slow wave fronts. In order to do this we assume that the wave originates from a point source. In this point the phase speed in all directions is calculated, supplying us with information of the wave front location in the next snapshot. For all subsequent snapshots we calculate the phase speed in each point of the previous wave front. The next fast (slow) wave front is then the outer edge of all fast-wave quasicircles (slow-wave double quasicircles).

We let our theoretical wave fronts for both fast and slow waves propagate from two point sources at the bottom of the domain: one at the pore axis at $(x, z)=(0,-0.095) \mathrm{Mm}$ and one at the edge of the driver at $(x, z)=(0.2,-0.095) \mathrm{Mm}$, starting from $t=0 \mathrm{~s}$. Figure 9 shows the saturated wave energy flux parallel (left) and perpendicular (right) to the magnetic field at $t=124$ $\mathrm{s}$. The theoretical fast wave fronts (solid lines) and slow wave fronts (dashed lines) are overplotted in green. By examining the time sequence, which is available as movies online, it is evident that there are many waves propagating with the exact same shape and speed as the theoretical fast wave fronts outside the pore. We therefore identify those waves as fast waves. They can be seen most clearly in Figure 9 between $x \approx 0.7 \mathrm{Mm}$ and $x \approx 1.7 \mathrm{Mm}$.

There are also waves propagating out of the pore with the same shape and speed as the theoretical slow wave fronts. We therefore identify these waves as slow waves. In Figure 9 they can be predominantly seen in the perpendicular flux component between the two dashed lines. When observing the time sequence for the parallel component and focusing on that region an interaction between fast and slow waves can be seen. However, at this point the flux has already exited the pore, and we thus do not discuss this further.

When observing the full time sequence of the movies of Figure 9, the theoretical wave fronts eventually develop a dip close to the border of the pore (e.g., snapshot 35). This is especially prominent for the fast waves, and is also seen in the simulation data. The reason for this dip is the density structure at that location, which can be seen in Figure 4. The difference in density leads to a difference in phase speed.

Although there are clearly waves leaking out of the pore, most of the flux is contained within the pore, following the magnetic field lines. To estimate the effect of lateral leakage on the damping of energy flux with height, we compare the timeintegrated total flux present along the field line highlighted in blue in Figures 7 and 9 (which is the total flux lost laterally) with the time-integrated total flux inside the pore at the bottom of the domain (which is the total incoming flux). The time integration of the flux is calculated for the first wave front over one period $T$ for all locations

$$
\boldsymbol{E}_{\boldsymbol{t}}(x, z)=\int_{t_{1}(z)}^{t_{2}(z)} \boldsymbol{E}(x, z, t) d t
$$



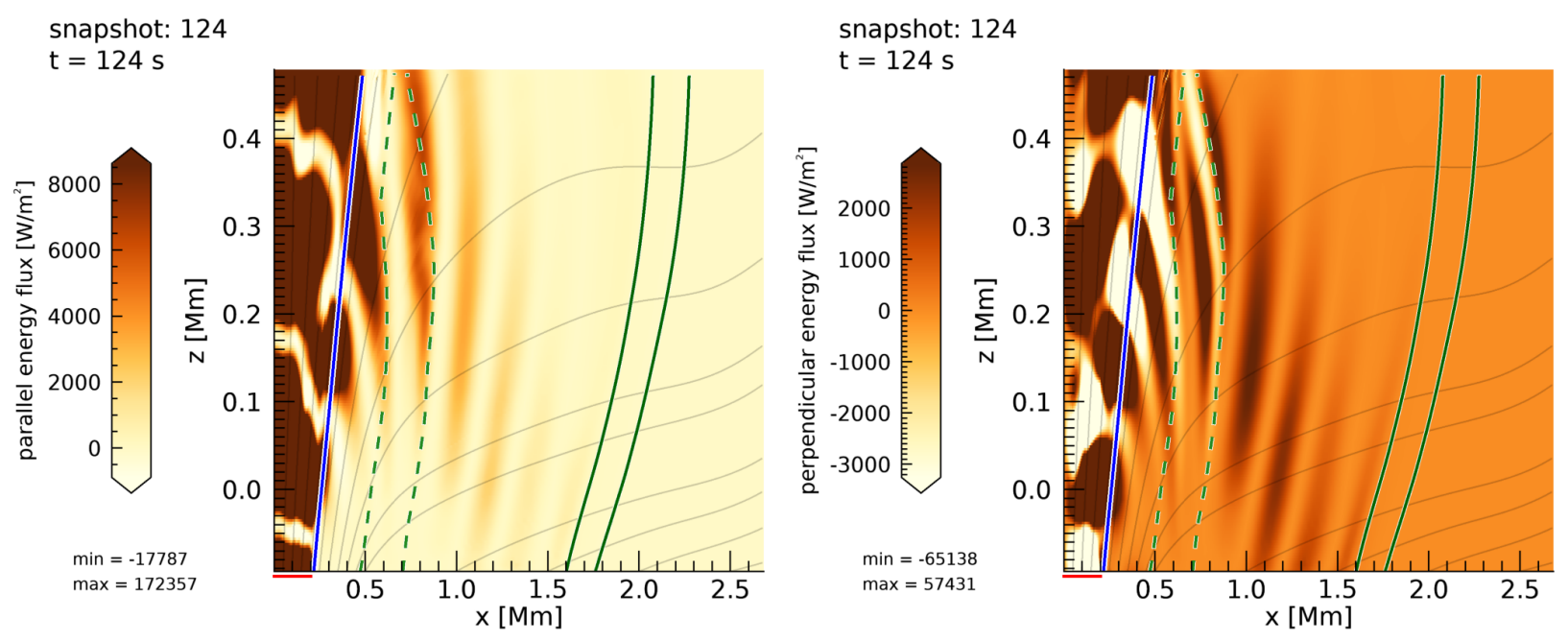

Fig. 9. Snapshot of the wave energy flux parallel (left) and perpendicular (right) with respect to the magnetic field. The color range is saturated. The solid (dashed) green lines show the first theoretical wave fronts of the fast (slow) waves; the gray lines show the magnetic field lines. The red bars below the $x$-axis indicate the driver location. The blue lines highlight the field line considered for the analysis in Figure 8 . Movies of the full time sequence are available online.

where $\boldsymbol{E}$ is the wave energy flux according to Equation 11 and $t_{1}(z)$ and $t_{2}(z)$ is the time of the beginning and end, respectively, of the first wave front at height $z$, with $t_{2}(z)=t_{1}(z)+T$.

For the calculation of the escaped flux we chose a field line rooted slightly outside the driver region in order to be sure that all flux at that location has exited the pore. We then integrate the time-integrated flux components along this field line from the root of the field line at the bottom of the domain until height $z$, before calculating the time-integrated total flux. The total escaped flux is then

$E_{t, \mathrm{esc}}(z)=\left(\left[\int_{0}^{l(z)} E_{t, \|}(x, z) d l\right]^{2}+\left[\int_{0}^{l(z)} E_{t, \perp}(x, z) d l\right]^{2}\right)^{1 / 2}$,

where $l(z)$ is the length of the field line at height $z$ and the integrals of the fluxes are taken along the field line. Here $E_{t, \|}$ and $E_{t, \perp}$ are the parallel and perpendicular components of the timeintegrated energy flux (Equation 14) with respect to the magnetic field (and therefore the field line), with $E_{t, \|} \perp E_{t, \perp}$. The integration is done before calculating the absolute value to allow flux with opposing signs to cancel out.

Similarly, the total flux contained in the pore at the bottom of the domain is calculated by

$E_{t, \mathrm{bot}}=\left(\left[\int_{0}^{x_{l}} E_{t, x}\left(x, z=z_{\mathrm{bot}}\right) d x\right]^{2}+\left[\int_{0}^{x_{l}} E_{t, z}\left(x, z=z_{\mathrm{bot}}\right) d x\right]^{2}\right)^{1 / 2}$,

where $x_{l}=0.22 \mathrm{Mm}$ is the $x$-position of the field line root, $z_{\mathrm{bot}}=$ $-0.095 \mathrm{Mm}$ is the $z$-location of the bottom of the domain, and the integrals are taken horizontally across the pore at the bottom of the domain. Here $E_{t, x}$ and $E_{t, z}$ are the $x$ - and $z$-components of the time-integrated energy flux, with $E_{t, x} \perp E_{t, z}$. by

The effect of wave leakage on the damping is then estimated

$e_{\text {damp }}(z)=1-\frac{E_{t, \text { esc }}(z)}{E_{t, \text { bot }}}$.

The result of Equation 17 is shown in Figure 8 (right) as the blue dashed line. There is a significant difference between this line and the line showing missing flux when only considering geometric spreading (orange dash-dotted line). Both methods are estimates, and we expect the actual effect of lateral wave leakage to lie between these lines.

\section{Conclusions and discussion}

We created a MHS model close to equilibrium, which was inspired by observational data of a solar pore (GM21) and investigated possible damping mechanisms by driving the model with a vertical velocity perturbation at the bottom of the domain. We found that, even if viscosity, resistivity, or thermal conduction are included, the strong damping from the observations could not be reproduced at all by using a driver that covers the whole bottom boundary. When switching to a localized driver, however, the results show strong damping in our simulations. This damping occurs because of a) geometric spreading, where the flux is spread over a wider area due to diverging field lines and b) lateral wave leakage, where waves leave the pore. Therefore, even if only considering classic wave effects, significant damping can be achieved. Wave leakage at the edge of a solar pore was indeed already observed by Stangalini et al. (2011).

\subsection{Effects of differences between observed pore and model \& comparison of simulations to observations}

It was mentioned in Section 2.2 there are differences between our model and the observational data example pore (GM21, pore $3)$. The differences in density and pressure profiles mainly lead to differences in characteristic wave speeds. This does not affect the damping due to geometric spreading, as this damping mechanism is only dependent on the magnetic field structure, which is similar to the observations, with nearly vertical inclination inside the pore and nearly horizontal field lines outside.

An important point we have to note, however, is the sound speed profile, as shown in Figure 10. In our model, the sound speed generally increases with height, whereas it is the opposite for the observations. In addition, there is a strong horizontal structuring, with lower speeds at the center and the border of the pore. From applying Snell's refraction law, as also discussed in 


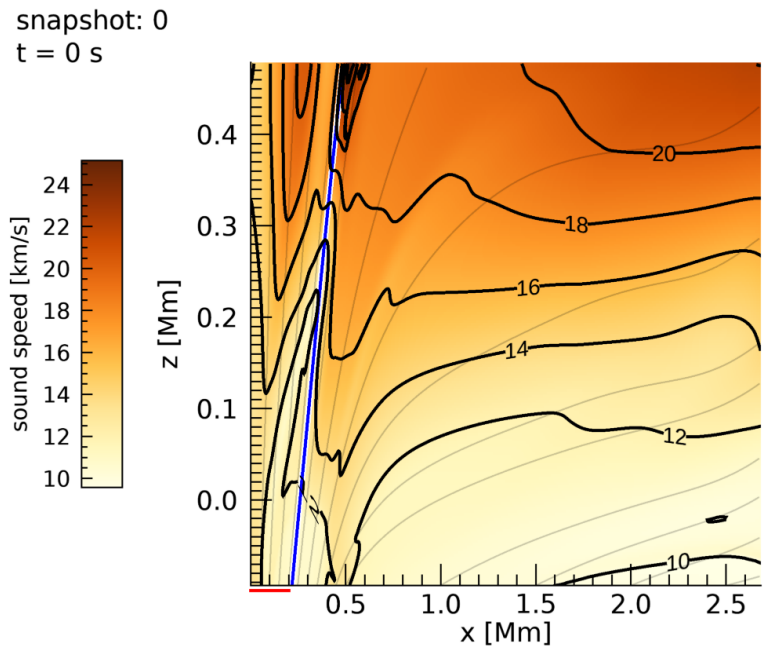

Fig. 10. Sound speed of the initial atmosphere. Gray lines show magnetic field lines. The red bar below the $x$-axis indicates the driver location for simulations with localized driver. The blue line highlights the field line considered for the analysis in Figure 8 Contours for the sound speed are shown in thick black lines.

the context of sunspots by Khomenko \& Collados (2006), we know that waves travelling into a medium with higher phase speed refract away from the line perpendicular to the constantphase-speed-line. If in our simulations the fast (acoustic) waves are propagating along the diverging field lines, they are refracted away from the pore. Therefore, should the fast lateral waves in our simulations exclusively occur because of refraction, we would not expect acoustic waves escaping laterally for the observations of pores like in GM21. The effect of lateral leaking for magnetic waves should be the same, however, as the Alfvén speed profile in our simulations is similar to the observations.

Evidence of at least some fast wave refraction occurring in our simulations is seen in the amplitude of the wave energy flux, where the amplitude is increased at the center and the boundary of the pore compared to the region in between. Those regions coincide with regions of lower sound speeds and waves are therefore refracted toward those regions. The increased amplitude in the pore boundary therefore does not occur because of sausage surface waves. Since the sound speed is higher at the location just outside the pore, waves that are located outside the pore would be refracted into the pore. This could be one of the reasons why the energy flux profile increases with height for the full driver (Figure 6), as there are ample waves present outside the pore to be refracted. In addition, fast wave energy flux that escaped from the pore was eventually refracted down toward the bottom of the domain in the simulations with localized driver. This can be seen in Figure 9 (right), where the perpendicular flux component for the fast waves outside the pore is mainly positive and therefore directed downward, considering the nearly horizontal field lines. This refraction of fast waves is similar to what was found by Khomenko \& Collados (2006).

The observations of pore 3 GM21 also show higher energy flux concentrations at the pore boundaries. Contrary to the events in our simulations, it was found that these flux concentrations are due to surface sausage modes. This could possibly promote additional lateral wave leakage as flux already present at the edge of the pore could more easily escape.

A crucial difference between observations and simulations is that due to the cadence of the instruments, GM21 were only able to investigate slow waves, whereas in this paper we have a combination of slow and fast waves. By splitting the energy flux into magnetic (Poynting) and hydrostatic contributions, slow and fast waves could have been studied separately. However, most of the slow waves in our simulations with localized driver stem from the sharp edge of the step-function, causing most of the slow waves being concentrated just inside and atop the considered field line marking the boundary of the pore in our analysis (blue highlighted field line in e.g. Figure 9), with little slow wave flux inside the rest of the pore. We therefore only considered the total flux for our analysis, as our estimate for the influence of damping due to lateral wave leakage (Equation 17) would not have worked for slow waves alone. On the other hand, there was no need to exclude slow waves from the same analysis as the magnitude of the Poynting flux is about three orders of magnitude smaller than the hydrostatic component.

In our model $\beta>1$ everywhere, whereas $\beta<1$ is expected inside the pores according to the observations. This basically means that the fast waves inside the pore in this paper correspond to the slow waves observed in GM21 as they both have predominantly acoustic properties. While slow waves are allowed to propagate in all directions except directly perpendicular to the magnetic field (see Equation 13, their phase speed as a function of angle to the magnetic field has a different shape than for fast waves. While, according to our results, slow waves also leave the pore, it is possible that due to this different shape fewer low $\beta$ slow acoustic waves (observations) would leave the pore than fast acoustic waves in our simulations. However, the slow acoustic waves in observations are still comparable to the fast acoustic waves simulated here. It is therefore reasonable to assume that within the pore the slow wave energy flux would be dominant over the fast wave energy flux if our model atmosphere had $\beta<1$ in that region. Applying this assumption to the real world highlights one of the difficulties in observing fast modes: the fast wave flux would be overshadowed by the slow wave flux. In addition, having a low plasma- $\beta$ inside the pore inevitably leads to a $\beta=1$ (or $v_{s}=v_{A}$ ) layer at the border of the pore with high $\beta$ outside. In these layers waves are strongly subjected to mode conversion (Cally 2005, 2006, Schunker \& Cally 2006, Hansen et al. 2015). Whether these mode conversions increase the amount of energy flux escaping from the pore or have a channeling effect in the pore will have to be determined in future work.

\subsection{On other limitations of the current study}

In this work, we did not account for any radiative losses. According to Carlsson \& Stein (2002), acoustic waves in the photosphere are much more damped at higher frequencies, meaning that the impact of this damping mechanism in our simulations would be larger than for the observations of GM21, who observe longer periods.

Our simulations were done on a 2D Cartesian grid. In 2D, the "area" inside the pore at each height is just a 1D line. Therefore, we estimated the damping due to geometric spreading to be proportional to $1 / R(z)$ with $R(z)$ the distance between the pore axis and a field line. In $3 \mathrm{D}$, however, we expect the wave energy flux due to this effect to decrease with $1 / R(z)^{2}$. Estimating the change in effect from $2 \mathrm{D}$ to $3 \mathrm{D}$ for wave leakage is more difficult. We assume that it is dependent on the ratio of the area inside the pore to the area that has been available for flux to escape, which is the mantle of the pore up to a specific height. This ratio is $R(z) / l(z)$ in $2 \mathrm{D}$ and $R(z)^{2} \pi /(2 R(z) \pi l(z))$ in $3 \mathrm{D}$, with $l(z)$ describing the length of the considered field line from the root up to a certain height $z$. Therefore, the dependence $R(z) / l(z)$ can also be assumed for 3D. 
The increase in efficiency of geometric spreading for 3D could account for the difference between the damping in our simulations and the observed damping. We note that by assuming a 2D geometry in our simulations we have excluded the possibility of Alfvén waves.

\subsection{Concluding remarks and future work}

As discussed above, there are both slow and fast waves present in our simulations. The slow waves are predominantly excited at the edge of the step-function driver. Simulations using a Gaussian-shaped driver instead show that slow waves are excited at the flank of the Gaussian, mostly at the steepest location. This leads to the conclusion that any kind of localized vertical driver would excite both slow and fast waves. Therefore, we also expect both kinds of waves to be present in the photosphere at all times. While slow modes have been observed in the photosphere many times, temporal resolution has so far limited similar studies for fast waves. However, future instruments on the Daniel K. Inouye Solar Telescope (DKIST), European Solar Telescope (EST), and National Large Solar Telescope (NLST) might provide the cadence needed to observe fast waves propagating at an inclined angle with respect to the magnetic field.

Observing the leaking waves as seen in our simulations might be challenging as the magnitude of the vertical (line-ofsight) velocity perturbations is roughly a factor of ten lower than the perturbations inside the pore. However, since the wave fronts of the leaking waves are inclined from the vertical (as seen in Figure 9), an observer from above would see the integrated effects of waves in different phases (i.e., positive and negative velocities within the same pixel). This would lead to spectral line broadening. The possibility to observe the leaking waves using this effect can be investigated using forward modeling techniques, such as the FoMo code developed by Van Doorsselaere et al. (2016).

Acknowledgements. The authors thank the referee for their constructive comments. JMR and TVD have received funding from the European Research Council (ERC) under the European Union's Horizon 2020 research and innovation programme (grant agreement No. 724326). CAG-M, DBJ, and SDTG are grateful to Invest NI and Randox Laboratories Ltd. for the award of a Research \& Development Grant (059RDEN-1) that allowed the research framework employed to be developed. DBJ and SDTG also wish to acknowledge the UK Science and Technology Facilities Council (STFC) for funding under the Consolidated Grant ST/T00021X/1.

\section{References}

Balthasar, H., Collados, M., \& Muglach, K. 2000, Astronomische Nachrichten, 321,121

Bogdan, T. J., Carlsson, M., Hansteen, V. H., et al. 2003, ApJ, 599, 626

Borrero, J. M., Pastor Yabar, A., Rempel, M., \& Ruiz Cobo, B. 2019, A\&A, 632, A111

Botha, G. J. J., Arber, T. D., Nakariakov, V. M., \& Zhugzhda, Y. D. 2011, ApJ, 728,84

Cally, P. S. 1986, Sol. Phys., 103, 277

Cally, P. S. 2001, ApJ, 548, 473

Cally, P. S. 2005, MNRAS, 358, 353

Cally, P. S. 2006, Philosophical Transactions of the Royal Society of London Series A, 364, 333

Cally, P. S. \& Khomenko, E. 2019, ApJ, 885, 58

Cameron, R., Schüssler, M., Vögler, A., \& Zakharov, V. 2007, A\&A, 474, 261

Carlsson, M. \& Stein, R. F. 1997, ApJ, 481, 500

Carlsson, M. \& Stein, R. F. 2002, in ESA Special Publication, Vol. 505, SOLMAG 2002. Proceedings of the Magnetic Coupling of the Solar Atmosphere Euroconference, ed. H. Sawaya-Lacoste, 293-300

Centeno, R., Collados, M., \& Trujillo Bueno, J. 2006, ApJ, 640, 1153

Chen, S.-X., Li, B., Shi, M., \& Yu, H. 2018, ApJ, 868, 5

Cho, K. S., Bong, S. C., Chae, J., et al. 2013, Sol. Phys., 288, 23
Dedner, A., Kemm, F., Kröner, D., et al. 2002, Journal of Computational Physics, 175,645

Dorotovič, I., Erdélyi, R., Freij, N., Karlovský, V., \& Márquez, I. 2014, A\&A, 563, A12

Felipe, T., Kuckein, C., González Manrique, S. J., Milic, I., \& Sangeetha, C. R. 2020, ApJ, 900, L29

Felipe, T., Kuckein, C., \& Thaler, I. 2018, A\&A, 617, A39

Fujimura, D. \& Tsuneta, S. 2009, ApJ, 702, 1443

Garcia de La Rosa, J. I. 1987, Sol. Phys., 112, 49

Geeraerts, M., Van Doorsselaere, T., Chen, S.-X., \& Li, B. 2020, ApJ, 897, 120

Gilchrist-Millar, C. A., Jess, D. B., Grant, S. D. T., et al. 2021, Philosophical Transactions of the Royal Society of London Series A, 379, 20200172

Goedbloed, J., Keppens, R., \& Poedts, S. 2019, Magnetohydrodynamics of Laboratory and Astrophysical Plasmas (Cambridge University Press; Cambridge)

Goedbloed, J. P. H. \& Poedts, S. 2004, Principles of Magnetohydrodynamics: With Applications to Laboratory and Astrophysical Plasmas (Cambridge University Press)

Grant, S. D. T., Jess, D. B., Moreels, M. G., et al. 2015, ApJ, 806, 132

Grant, S. D. T., Jess, D. B., Zaqarashvili, T. V., et al. 2018, Nature Physics, 14, 480

Hansen, S. C., Cally, P. S., \& Donea, A.-C. 2015, Monthly Notices of the Royal Astronomical Society, 456, 1826

Jaeggli, S. A., Lin, H., Mickey, D. L., et al. 2010, Mem. Soc. Astron. Italiana, 81,763

Jess, D. B., Morton, R. J., Verth, G., et al. 2015, Space Sci. Rev., 190, 103

Jess, D. B., Reznikova, V. E., Van Doorsselaere, T., Keys, P. H., \& Mackay, D. H. 2013, ApJ, 779, 168

Jess, D. B., Snow, B., Houston, S. J., et al. 2020, Nature Astronomy, 4, 220

Kato, Y., Steiner, O., Hansteen, V., et al. 2016, ApJ, 827, 7

Keys, P. H., Morton, R. J., Jess, D. B., et al. 2018, ApJ, 857, 28

Khomenko, E. \& Cally, P. S. 2019, ApJ, 883, 179

Khomenko, E. \& Collados, M. 2006, ApJ, 653, 739

Krishna Prasad, S., Jess, D. B., Van Doorsselaere, T., et al. 2017, ApJ, 847, 5

Lamb, H. 1909, Proceedings of the London Mathematical Society, s2-7, 122

Maltby, P., Avrett, E. H., Carlsson, M., et al. 1986, ApJ, 306, 284

Mignone, A., Bodo, G., Massaglia, S., et al. 2007, ApJS, 170, 228

Mignone, A., Tzeferacos, P., \& Bodo, G. 2010, Journal of Computational Physics, 229, 5896

Moreels, M. G., Goossens, M., \& Van Doorsselaere, T. 2013, A\&A, 555, A75

Morton, R. J., Erdélyi, R., Jess, D. B., \& Mathioudakis, M. 2011, ApJ, 729, L18

Morton, R. J., Verth, G., Jess, D. B., et al. 2012, Nature Communications, 3, 1315

Nakariakov, V. M. \& Zimovets, I. V. 2011, ApJ, 730, L27

Ossendrijver, M. 2003, A\&A Rev., 11, 287

Riedl, J. M., Van Doorsselaere, T., \& Santamaria, I. C. 2019, A\&A, 625, A144

Roberts, B. 2006, Philosophical Transactions of the Royal Society of London Series A, 364, 447

Ruiz Cobo, B. \& del Toro Iniesta, J. C. 1992, ApJ, 398, 375

Schmitz, F. \& Fleck, B. 1998, A\&A, 337, 487

Schunker, H. \& Cally, P. S. 2006, MNRAS, 372, 551

Sobotka, M. 2003, Astronomische Nachrichten, 324, 369

Stangalini, M., Del Moro, D., Berrilli, F., \& Jefferies, S. M. 2011, A\&A, 534, A65

Thomas, J. H. \& Weiss, N. O. 2004, ARA\&A, 42, 517

Van Doorsselaere, T., Antolin, P., Yuan, D., Reznikova, V., \& Magyar, N. 2016, Frontiers in Astronomy and Space Sciences, 3, 4

Yu, D. J., Van Doorsselaere, T., \& Goossens, M. 2017, A\&A, 602, A108

Article number, page 10 of 12 


\section{Appendix A: Simulations with driver below the cutoff frequency}

In order to focus on propagating waves all simulations in this paper have so far been conducted with a driver frequency well above the expected cutoff frequency of the model atmosphere, and therefore also with a period much smaller than the fiveminute waves observed by GM21. This means that for the current study, all effects of the cutoff frequency have been ignored. However, as mentioned in Section 1, even if the waves of GM21 definitely have a propagating character, they might be altered to partly evanescent waves by the existence of the cutoff frequency (Centeno et al. 2006). In this section we explore the possibility of damping due to evanescent waves by conducting the same two experiments as before, namely simulations with full driver and localized driver, but with a lower driver frequency.

\section{Appendix A.1: Cutoff frequency and new driver period}

It is commonly accepted that acoustic waves with frequencies below the cutoff frequency are not allowed to propagate, but are standing and evanescent. However, it is difficult to define an exact value for the cutoff frequency, and numerous different definitions exist. Centeno et al. (2006) show that when radiative losses are involved there is no clear cutoff frequency that distinguishes between fully propagating or fully evanescent waves. Felipe et al. (2018) compared analytical definitions for the cutoff frequency suitable for sunspot umbrae from Lamb (1909), Schmitz \& Fleck (1998), and Roberts (2006) to the observed cutoff. The results generally agree. Using the same analytical expressions as discussed in Felipe et al. (2018) on the observational data obtained by GM21 for pore 3 shows that waves with a period of five minutes indeed have a lower frequency than the cutoff frequency for at least most of the observed domain.

According to the same equations, a driver period of five minutes would still result in a frequency above the cutoff frequency for our model atmosphere. To mimic the conditions of the observations, we choose a longer driver period of $T=7$ minutes for the following simulations. To include at least one full period of the driver the simulations are run for 500 seconds.

\section{Appendix A.2: Results}

Figure A.1 shows the height-time graph of the wave energy flux parallel to the magnetic field at the axis of the pore for the simulation with localized driver. The characteristic speeds (starting from steepest: fast speed $v_{\mathrm{fa}}(\theta=\pi / 2)=\left(v_{A}^{2}+v_{s}^{2}\right)^{1 / 2}$, sound speed, Alfvén speed, cusp speed) are plotted as black lines, while the contour at value zero is shown in red. The initial part of the first wave (i.e., the initial disturbance where the flux is above zero for the first time) propagates with the sound speed (black dashed line overplotted on first red line) as it did for the propagating waves in Section 3 Then, however, the waves get altered by the effects of the cutoff frequency to approximately standing waves within less than half a driver period, as can be seen from the nearly vertical features in the figure. This is not what was observed in GM21, who found clear evidence of propagating waves. The difference might be accounted for by the neglect of radiative losses in our simulations.

We performed the same study for the wave energy flux damping as in Sections 3.1 and 3.2, but for the low-frequency driver. Figure A.2 shows the results for the full driver, while Figure A.3 shows the results for the localized driver. It is immediately apparent that the energy flux for the full driver is now heavily damped

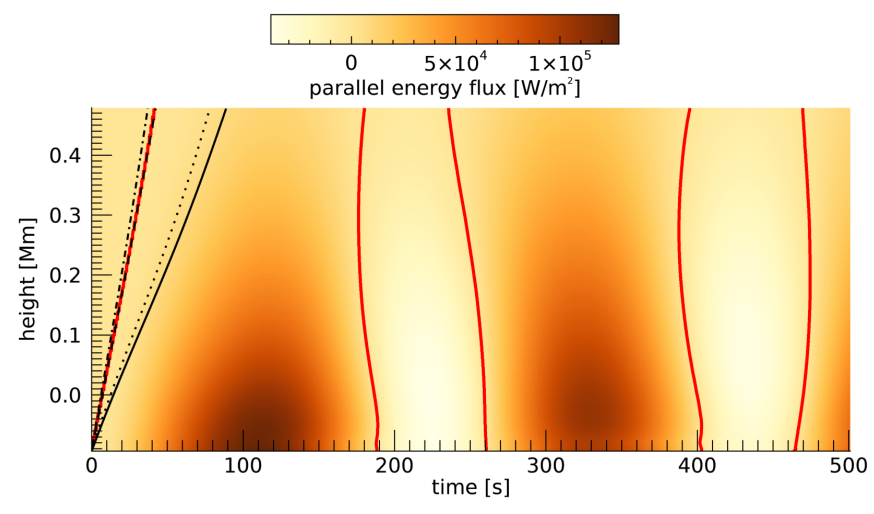

Fig. A.1. Parallel wave energy flux as a function of height and time at the pore axis for the simulation with localized driver with a period of 7 minutes. The black lines show (from steepest to flattest) the fast speed (dash-dotted line), sound speed (dashed line), Alfvén speed (dotted line), and cusp speed (solid line). The red lines show the contours for zero flux. The frequency of the energy flux is approximately doubled compared to the driver period because of phase difference between $p^{\prime}$ and $v^{\prime}$ (see Equation 11 .

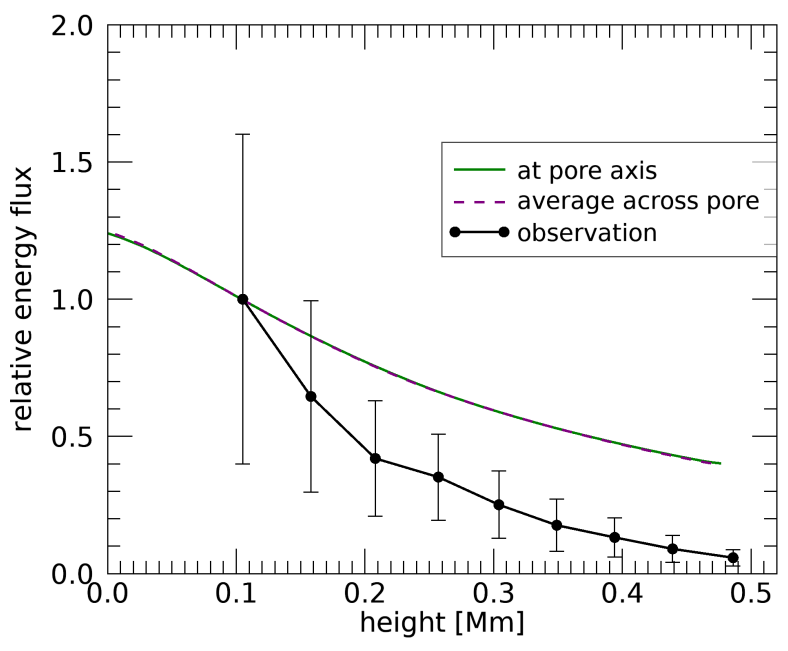

Fig. A.2. Same as Figure 6 but for a driver period of 7 minutes.

as well, about the same amount as the energy flux for the localized high-frequency driver (Figure 8). The energy flux for the localized low-frequency driver (Figure A.3 is damped even more, probably because the damping with height is not decreased by inward refracted waves as for the full driver.

\section{Appendix A.3: Discussion}

It is obvious that the choice of driver frequency strongly affects the damping in our simulations. However, whether this is purely due to evanescent waves is not fully clear.

On the one hand, the dash-dotted orange curve in Figure A.3. which shows the damping without effect of geometric spreading, strongly follows the solid green line, which is the full damping in our simulation with the localized low-frequency driver. This hints that geometric spreading has little to no effect in this case. At the same time the dashed blue line, which is an estimate for the influence of lateral leakage, is nearly constant, meaning that this effect is also not very strong. Therefore, a crucial damping mechanism is missing, which is likely the reflection of waves due to the cutoff frequency. 


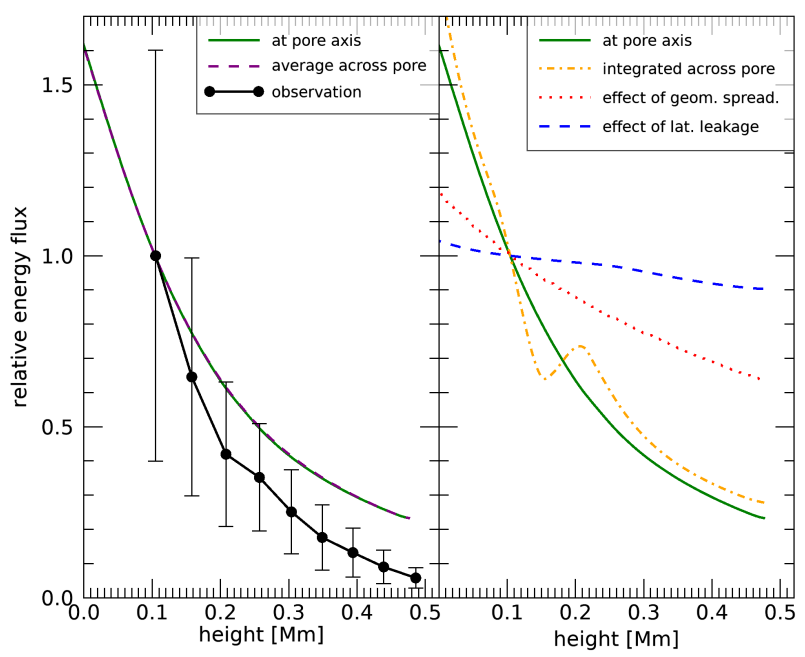

Fig. A.3. Same as Figure 8 , but for a driver period of 7 minutes.

On the other hand, these new simulations and their analysis are subject to some limiting factors. First of all, due to the low frequency, the wavelengths of the resulting waves are significantly longer than the size of the computational domain. This could lead to strange boundary effects influencing the results. Since the ratio of the wavelength to the size of the pore (which is smaller in our model than in the observations) also changes drastically, this could account for the decreased effects of geometric damping and lateral wave leakage. In addition, due to the waves starting at some final time $t_{0}$, there are no waves present in the domain before the first waves reach a certain height (i.e., left of the first red line in Figure A.1. Therefore, when integrating the wave energy flux over time, the lower integration boundary $t_{1}(z)$ was chosen by using a relative threshold to determine the onset of the first wave at every height. This line basically coincides with the sound speed line (dashed) in Figure A.1 The upper integration boundary was then determined by $t_{2}(z)=t_{1}(z)+T$, with $T$ being the driver period. Effectively, the time integration for the simulations with high-frequency driver was done over the first period of the wave, as a translation of $t_{1}(z)$ by $T=30 \mathrm{~s}$ resulted in a $t_{2}(z)$ being located right in front of the next wave train. This is not the case for the low-frequency waves because they change from propagating to standing waves within the first wave period, meaning that their steepness changes in Figure A.1. Therefore, it is not clear over which time period the integration should be performed, and the choice might affect the shape of the damping curves in Figures A.2 and A.3

Moreover, even if the limitations listed above have little to no effect, there are still no propagating waves in our low-frequency simulations, as opposed to the observations of GM21. Therefore, the damping in the low-frequency simulations due to evanescent waves is expected to be much stronger than for the observations, where the waves were at least partly propagating. This validates the study of the other damping mechanisms presented in this paper. 